REVIEW

\title{
Diagnosis of tuberculous aetiology in pericardial effusions
}

\author{
G Cherian
}

Postgrad Med J 2004;80:262-266. doi: 10.1136/pgmi.2003.013664

The diagnosis of tuberculous aetiology in pericardial effusions is important since the prognosis is excellent with specific treatment. The clinical features may not be distinctive and the diagnosis could be missed particularly with tamponade. With the spread of HIV infection the incidence has increased. The diagnosis largely depends on histopathology of the pericardial tissue or culture of Mycobacterium tuberculosis from this tissue or fluid, but patients without haemodynamic compromise do not require pericardiocentesis. Histopathology may, however, show non-specific findings in a significant number. This review is an update on the diagnostic difficulties, current research, and criteria for diagnosis.
Correspondence to: Professor George Cherian, Narayana Hrudayalaya, 258/A-Bommasandra Industrial Area, Anekal Taluk, Bangalore 562 158, India; gcherian@vsnl.net

Submitted 18 August 2003 Accepted 26 October 2003
$\mathrm{T}$ uberculous pericardial effusion (TPE) is still common in African and Asian countries, ${ }^{12}$ but with migrant populations and the spread of AIDS $^{3}$ there are now more frequent reports from other parts of the world like Europe and the Western hemisphere. ${ }^{45}$ TPE usually presents as a slowly progressive febrile illness. When it presents as an acute pericarditis, which is uncommon, or as cardiac tamponade, ${ }^{2}$ which is frequent, the diagnosis is more likely to be delayed or missed. The delay from hospital admission to diagnosis was 5.2 weeks in a report from Spain $^{6}$ and in another from the USA the diagnosis was first made only at necropsy in $17 \%$ of patients. ${ }^{7}$ Malignant, viral, and chronic idiopathic effusions are some of the other causes of large effusions. Effusions associated with malignancy are usually but not always apparent at presentation. Chronic idiopathic effusions in which no aetiology could be established are a common cause of tamponade even in countries like the UK, France, and the USA with a reported incidence varying from $11 \%-32 \%{ }^{8-10}$

The diagnosis of tuberculosis as the aetiological cause is important. Without specific treatment the average survival was 3.7 months in a report from Africa and only 4/20 (20\%) were alive at six months. ${ }^{1}$ On the other hand the prognosis with appropriate medical treatment is excellent as seen in recent reports. ${ }^{11}{ }^{12}$ The confirmation of tuberculous aetiology currently depends on histopathological study of the pericardium, the demonstration of tubercle bacilli in the pericardial tissue or pericardial fluid, the presence of proved tuberculosis elsewhere in the body, or the response to specfic treatment. ${ }^{13}$ Histopathological examination, however, can at times give non-specific findings even when tubercle bacilli have been cultured from the pericardial fluid. ${ }^{14}$

\section{PATHOGENESIS OF TUBERCULOUS PERICARDITIS}

There are many ways in which the pericardium may be involved in tuberculosis. In a rare case there may be direct spread from tuberculous pneumonia. The pericardium can be seeded in miliary tuberculosis and in such instances other organ systems dominate the presentation. Direct extension from an infected visceral pleura or rib is rare. Most often the spread is from the breakdown of infection in mediastinal nodes directly into the pericardium and particularly those at the tracheobronchial bifurcation. ${ }^{15}$ The spread is over lymph channels that merge at points where the parietal pericardium and the pleura separate.

\section{LYMPHATIC DRAINAGE OF THE PERICARDIUM}

Studies in human cadavers, ${ }^{16}$ in the macaque monkey, ${ }^{17}$ and in the $\operatorname{dog}^{18}$ show that the lymphatic drainage of the pericardium is mainly to the anterior mediastinal, tracheobronchial, lateropericardial, and posterior mediastinal (juxta oesophageal) lymph nodes and not into the hilar nodes. This has an important bearing on the groups of mediastinal lymph nodes that are enlarged in TPE. The mediastinal node enlargement in TPE does not show up on routine chest radiographs (fig 1) but can be seen only on chest computed tomography or magnetic resonance imaging (MRI) studies. ${ }^{12}$ In other conditions associated with pericardial effusion and mediastinal adenopathy like lymphomas, malignancy, and sarcoid hilar node involvement is prominent even though in sarcoidosis the pericardium is involved in fewer than $3 \%$ of patients. ${ }^{19}$

\section{MEDIASTINAL LYMPH NODES IN TPE}

Sir William Osler from his review of consecutive necropsies found 275 with tuberculosis of whom seven had pericarditis. He concluded that caseous mediastinal lymph nodes were the usual focus of pericardial involvement. ${ }^{20}$ In another report, at necropsy all patients with TPE had mediastinal adenopathy. ${ }^{7}$ The groups of glands involved were not described. In another study, right scalene node biopsy was performed in six patients with TPE and even though the node was not enlarged, all six had evidence of tuberculosis. $^{21}$

Abbreviations: MRI, magnetic resonance imaging; PCR, polymerase chain reaction; TPE, tuberculous pericardial effusion 


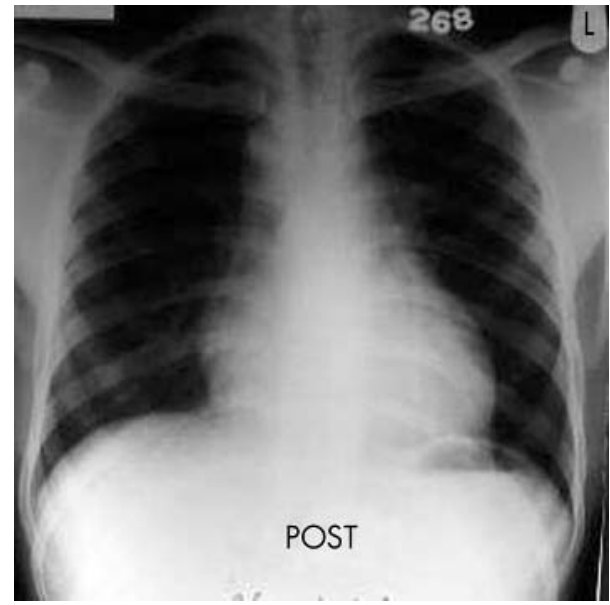

Figure 1 Chest radiograph after aspiration of same patient as in fig 4 taken at time of chest computed tomography. Nodes not seen on chest radiograph but seen only on chest computed tomogram.

\section{PLEURA AND PERIPHERAL LYMPH NODES IN TUBERCULOSIS}

Pleural effusion and peripheral lymphadenitis are two common forms of non-pulmonary tuberculosis. ${ }^{22}$ Rooney et al found that $50 \%$ of patients with TPE who were necropsied had pleural effusion due to tuberculous pleuritis. ${ }^{7}$ Tuberculous lymphadenopathy is characterised by caseation, which shows on computed tomographic studies as hypodense central areas, ${ }^{23}$ and "matting" on palpation, which is seen on the computed tomogram as coalescing of adjacent glands. The adenopathy clears or regresses on specific therapy. ${ }^{12} 24$

\section{RECENT DEVELOPMENTS IN THE DIAGNOSIS OF TPE Polymerase chain reaction (PCR)}

PCR technology has been used for nucleic acid amplification in the diagnosis of tuberculosis. ${ }^{25}$ Cegielski et al compared PCR, culture, and histopathology in the diagnosis of TPE. ${ }^{26}$ PCR was performed with both pericardial fluid and tissue with IS6110 based primers specific for the Mycobacterium tuberculosis complex. They concluded that the overall accuracy of PCR approached the results of conventional methods, although PCR was faster. The sensitivity for pericardial fluid was poor and false positive results with PCR remain a concern. ${ }^{27}$

\section{Serodiagnosis}

The potential for serodiagnosis of TPE has been reported by $\mathrm{Ng}$ et al who tested a solid phase antibody competition sandwich ELISA (SACT-CE) method. ${ }^{28}$ A monoclonal antibody (CDC/WHO reference number IT39) which was raised against a specific epitope on the $M$ tuberculosis $30 \mathrm{kDa}$ antigen was used. All but one of the patients had negative sputum microscopy for acid fast bacilli. A sensitivity of $61 \%$ (at $96 \%$ specificity) was achieved. It is unlikely that this technology will be widely applied. A more recent report suggests that there is a broad clonal heterogeneity of antigen specific CD4+T-cells localising at the site of disease during tuberculosis. ${ }^{29}$

\section{Adenosine deaminase and other markers}

Adenosine deaminase levels are believed to reflect T-cell activity. The levels with TPE have varied from 10-303 U/l in one report, ${ }^{30}$ and with a cut off level of $30 \mathrm{U} / \mathrm{l}$ the sensitivity was $94 \%$ and specificity $68 \%$ with a positive predictive accuracy of $80 \%$. In this study the median interferon-gamma concentration in TPE was $>1000 \mathrm{pg} / \mathrm{l}$ and significantly higher than malignancy or non-tuberculous effusions $(p<0.0005)$. A cut off value of $200 \mathrm{pg} / \mathrm{l}$ for interferon-gamma resulted in a sensitivity and specificity of $100 \%$ for the diagnosis of TPE. In another report the adenosine deaminase values were a mean (SD) of 126 (16.68) U/l and significantly higher than neoplastic, idiopathic, purulent bacterial, and radiotherapy groups. ${ }^{31}$ On the other hand it has been suggested adenosine deaminase and lysozome levels have to be taken into account in attempting an early diagnosis of TPE. ${ }^{32}$

\section{Nuclear imaging}

Gallium-67 and indium-111 scintigraphy have been used in the diagnosis of TPE. The results are, however, non-specific and there are other cardiac causes of gallium-67 uptake. ${ }^{33} 34$

\section{Chest computed tomography}

Recent observations on chest computed tomography regarding the detection of mediastinal lymph nodes in TPE and their value in diagnosis of TPE are discussed later. ${ }^{12}$ Computed tomography also allows study of the pericardium and the pleural changes (fig 2).

\section{EVALUATION OF TUBERCULOUS PERICARDIAL EFFUSION \\ Clinical features}

Asymptomatic presentation or small effusions are infrequent unless picked up during screening during the course of an illness like AIDS. The onset is most often insidious with loss of weight and fatigue, ${ }^{17}$ but could be more acute and occasionally explosive with tamponade. ${ }^{2}{ }^{12}$ The presenting symptoms appear to be similar all over the world. Fever (73\%-97\%) and dyspnoea $(80 \%-88 \%)$ are the most frequent symptoms while precordial pain $(39 \%-59 \%)$ is seen less often. ${ }^{1712}{ }^{15}$ Pericardial rub is present in $(37 \%-84 \%)^{17}$ and rarely can even be triphasic. ${ }^{12}$ Raised jugular venous pressure is frequent and pulsus paradoxus is present in $(23 \%-71 \%)$ with tamponade (box 1). ${ }^{15}$

Peripheral lymphadenopathy with matting which most often affects the cervical glands has been reported in 13\%$28 \%$ with $\mathrm{TPE}^{1}$ and biopsy of the gland is diagnostic. This is unlikely to be seen in Western countries as it is a late manifestation.

\section{Cardiac tamponade}

Cardiac tamponade is a common complication of TPE. Jain et al found that $60 \%$ of patients with tamponade had tuberculosis. $^{2}$ In another study of 44 children with TPE, $90 \%$ had tamponade. ${ }^{35}$ In a recent report from the USA both patients reported had tamponade ${ }^{36}$ and in an earlier report 6/11 deaths were due to tamponade. ${ }^{7}$ Since tamponade may

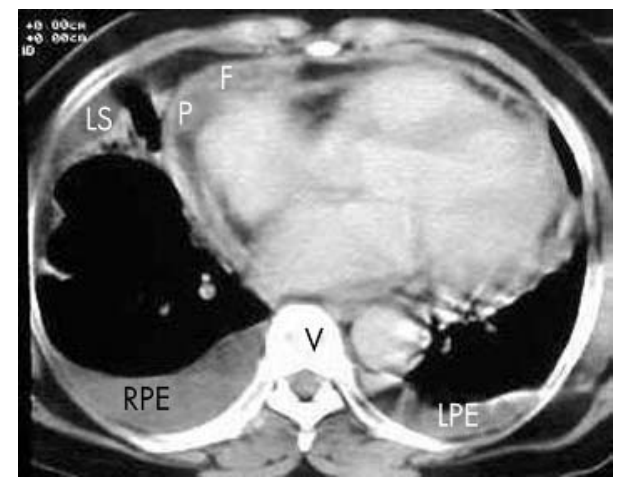

Figure 2 Pre-treatment chest computed tomogram after aspiration showing irregular pericardial thickening $(P)$ and some fluid $(F)$. Right (RPE) and left (LPE) pleural effusion with strands and loculation (LS) in pleural space. 


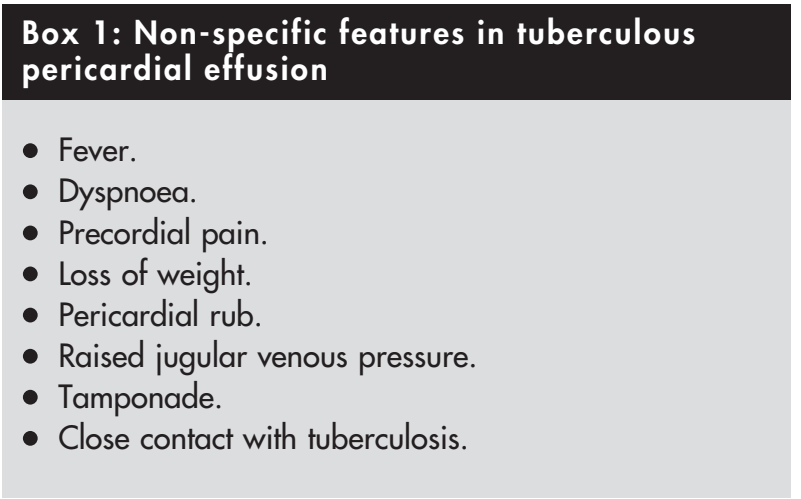

in part be due to late presentation , the incidence is likely to be higher in Afro-Asian countries. ${ }^{12}$

\section{HIV infection}

Tuberculosis has been reported as the predominant cause of large pericardial effusions in HIV infected patients in Tanzania. ${ }^{37}$ These patients were in an early stage of HIV infection and responded well to treatment. In an inner city hospital in the USA, out of 122 patients with pericardial effusion of varying sizes, 40 were HIV related and accounted for $20 \%$ with tamponade. ${ }^{3}$

\section{Chest radiography}

Apart from the signs of pericardial effusion, radiological evidence of pulmonary tuberculosis may be present. ${ }^{17}$ Pleural effusions are present in about $50 \%$ because of tuberculous pleuritis, ${ }^{7}$ and a tuberculous granuloma on pleural biopsy can help in a specific diagnosis.

\section{Chest computed tomography}

Chest computed tomography can yield valuable information in addition to the pleural changes (fig 2).The pericardium is irregular, not calcified, and is thickened measuring a mean (SD) of 4.4 (1.9) mm. ${ }^{12}$ Normal mediastinal lymph nodes are $<10 \mathrm{~mm}$ and do not change in size on follow up. ${ }^{38}{ }^{39}$ Enlarged mediastinal lymph nodes $>10 \mathrm{~mm}$ detected on chest computed tomography have been reported recently (figs 3 and 4 ) and for the first time in virtually $100 \%$ of patients with

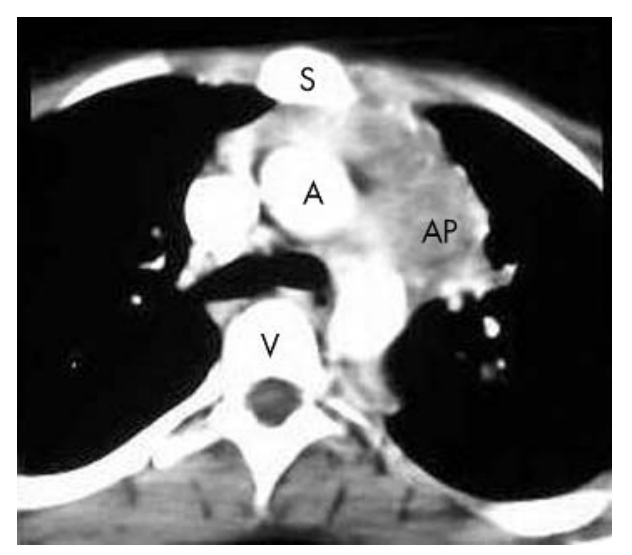

Figure 3 Pre-treatment chest computed tomogram after aspiration showing coalescing aortopulmonary (AP) nodes with matting and hypodense centre. S, sternum; $A$, aorta; $V$, vertebra. (Reprinted from Cherian G, Habashy AG, Uthaman B, et al. Detection and follow-up of mediastinal lymph node enlargement in tuberculous effusions using computed tomography. Am J Med 2003;1 14;319-22 with permission from Excerpta Medica, Inc).

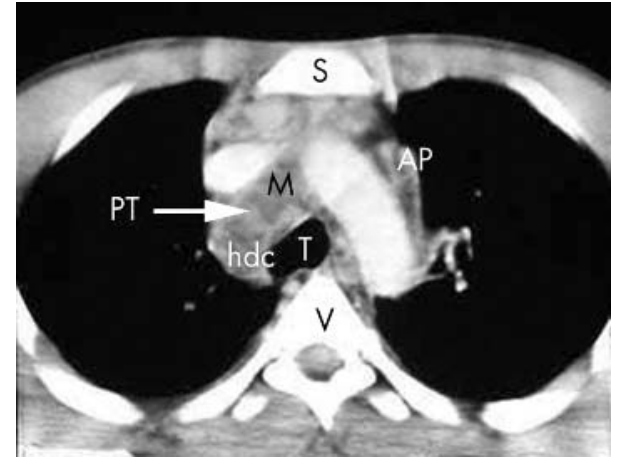

Figure 4 Pre-treatment chest computed tomogram of same patient as in fig 1 showing coalescing aortopulmonary (AP) and paratracheal (PT) nodes with matting $(M)$ and hypodense centre (hdc). S, sternum; $T$, trachea; $\mathrm{V}$, vertebra.

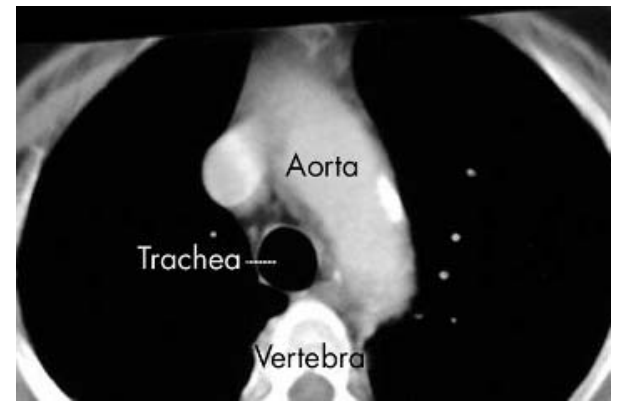

Figure 5 Chest computed tomogram in viral pericardial effusion after aspiration. No lymph nodes seen.

\section{Box 2: Features of mediastinal nodes in} tuberculous pericardial effusion

- Aortopulmonary, paratracheal, and carinal nodes most often involved.

- Typically coalesced (matted) with hypodense centre.

- Hilar nodes rare and inconspicuous.

- Hence nodes not seen on routine chest radiograph.

- Nodes seen only on chest computed tomography or MRI.

- Nodes disappear or regress on specific treatment.

TPE. ${ }^{12}$ They were found in all 22 patients with TPE and none of a control group with large viral/idiopathic or postoperative pericardial effusion (fig 5). The gland size varied from 10 $50 \mathrm{~mm}$, mean (SD) 19.5 (8.6), and the number of anatomical gland areas from $1-5$, mean (SD) 2.5 (1.22), with varying combinations. The aortopulmonary glands were most frequently enlarged (63\%) followed by the paratracheal (51.8\%), carinal $(40.7 \%)$, pretracheal $(25.9 \%)$, and hilar $(14.8 \%)$. The glands showed a hypodense centre in 52\%. Matting of adjacent glands was seen in $57.7 \%$. The hilar glands were least frequently enlarged which can be explained from the lymphatic drainage of the pericardium. The enlarged nodes could not be seen on the plain chest radiograph (fig l) and only on chest computed tomography and disappeared or regressed with specific anti tuberculous therapy. ${ }^{12}$ Features of mediastinal lymph nodes in TPE are shown in box 2 . 


\section{Echocardiography}

The pericardial exudate is thick and fibrinous with a tendency to form adhesions and in some instances constriction.On echocardiography there are patchy deposits 4-8 mm in thickness with "fibrinous" strands criss crossing the pericardial space. ${ }^{40}$ The appearance is quite characteristic.

\section{Tuberculin skin test}

The tuberculin skin test is done using the purfied protein derivative. A positive skin reaction is an induration $\geqslant 10 \mathrm{~mm}$ and a strongly positive response is defined as one $\geqslant 15 \mathrm{~mm}$ with or without excoriation of the skin. A positive tuberculin skin test has been found in all patients with TPE in one report $^{7}$ and in 239/240 in another. ${ }^{14}$ In the latter it was $>15 \mathrm{~mm}$ in $78 \%$. In a recent publication the induration measured 16.4 (3) mm. ${ }^{12}$ A strongly positive tuberculin skin test is of value when associated with tissue granuloma without acid fast bacilli or when typical non-hilar mediastinal adenopathy is detected on chest computed tomography.

\section{Pericardial fluid}

The greatest value of the pericardial fluid is when $M$ tuberculosis can be cultured as it is rare to find acid fast bacilli in a spun smear. The raised protein and lactic dehydrogenase values speak for an exudate. Lymphocytosis has been found in some studies ${ }^{41}$ but not in others. All these non-specific findings can be found in chronic idiopathic pericardial effusion as well. ${ }^{42}$ The value of adenosine deaminase and other markers has been discussed earlier.

\section{Culture of mycobacterium tuberculosis}

$M$ tuberculosis may be cultured from the sputum, tissue like the pericardium, pleura, scalene node, or other accessible enlarged nodes but such information is patchy. Recovery from the pericardial fluid has varied from $30 \%-100 \%$. Strang using special techniques was able to culture $M$ tuberculosis from all patients. ${ }^{43}$ The specimens were cultured in double strength Kirchner culture medium after bedside inoculation and also conventional culture in Stonebrink medium. $M$ tuberculosis was cultured in all 10 patients, in Kirchner medium in nine and conventional in one.

\section{Box 3: Criteria for diagnosis of tuberculous aetiology}

\section{Any of the following:}

Invasive

(1) Culture of $M$ tuberculosis from pericardial fluid or tissue.

(2) Pericardial tuberculous granuloma with acid fast bacilli.

(3) Pericardial tuberculous granuloma + positive tuberculin skin test.

(4) Pleural tuberculous granuloma with acid fast bacilli.

(5) Pleural tuberculous granuloma + positive tuberculin skin test.

(6) Tuberculous granuloma in scalene node or peripheral lymph node.

\section{Non-invasive}

(1) Active tuberculosis elsewhere in the body.

(2) Mediastinal (non-hilar)lymph nodes on chest computed tomography with hypodense centre and matting + positive tuberculin skin test (matting = coalescing of adjacent lymph nodes).

(3) Response to specific antituberculous therapy.
Box 4: Questions (answers at end of references)

(1) What are three common causes of large pericardial effusions which can pose difficulties in differential diagnosis?

(2) What is the most frequent route by which the pericardium is affected in tuberculosis?

(3) Which group of mediastinal nodes are least often enlarged in tuberculous pericardial effusion?

(4) Can mediastinal lymph nodes be detected both on a plain chest radiograph as well as a chest computed tomogram?

(5) Non-specific findings on histopatholoy of the pericardium may be found in around $30 \%$ of patients with proven tuberculous pericarditis. True or false?

(6) What is the prognosis of tuberculous pericardial effusion with specific treatment?

(7) Cardiac tamponade is a frequent mode of presentation with tuberculous pericardial effusion. True or false?

\section{Histological evidence}

Like recovery of $M$ tuberculosis from the pericardial tissue or fluid, histological evidence of a tuberculous granuloma with the demonstration of acid fast bacilli would be a definite diagnostic criterion. The typical granuloma is however not always found and the pericardial biopsy may show nonspecific findings even when $M$ tuberculosis is found in the pericardial fluid. Strang et al reporting on patients with TPE found that out of 49 patients in whom $M$ tuberculosis was recovered from the pericardial fluid the pericardial histological changes "characteristic of tuberculosis" was present only in 35/49 and 14/49 (29\%) had non-specific findings. ${ }^{14}$ In another study $2 / 19$ biopsies showed non specific findings. ${ }^{12}$ This difference may be due to the number of samples obtained at biopsy.

\section{Scalene node biopsy}

The report on scalene node biopsy has been discussed earlier. ${ }^{21}$ This would be a safe and relatively easy route to a specific diagnosis but so far there has been only one report.

\section{CONCLUSIONS}

Tuberculosis is an important cause of pericardial effusion in Afro-Asian countries and with the spread of HIV infection there is an increased incidence all over the world. Significant recent developments have been the use of PCR technology, ${ }^{26}$ improved techniques for the recovery of $M$ tuberculosis, ${ }^{43}$ observations on mediastinal lymph nodes on chest computed tomography, ${ }^{12}$ and more clearly defined observations on echocardiography. ${ }^{40}$ The diagnosis of tuberculous aetiology in pericardial effusions can be based on the criteria shown in box 3 .

\section{REFERENCES}

1 Desai HN. Tuberculous pericarditis. A review of 100 cases. S Afr Med J 1979;55:877-80.

2 Jain S, Sharma N, Varma S, et al. Profile of cardiac tamponade in the medical emergency ward of a North Indian hospital. Can J Cardiol 1999;15:671-5.

3 Chen Y, Brennessel D, Walters J, et al. Human immunodeficiency virusassociated pericardial effusion. A report of 40 cases and review of the literature. Am Heart J 1999;137:516-21.

4 Strang J. Tuberculous pericarditis. J Infect 1997;35:215-19.

5 Trautner BW, Darouiche RO. Tuberculous pericarditis: optimal diagnosis and management. Clin Infect Dis 2001;33:954-61.

6 Sagrista-Sauleda J, Permanyer-Miralda G, Soler-Soler J. Tuberculous pericarditis: ten year experience with a prospective protocol for diagnosis and treatment. J Am Coll Cardiol 1988;2:724-8.

7 Rooney JJ, Crocco JA, Lyons HA. Tuberculous pericarditis. Ann Intern Med 1970;72:73-8. 
8 Gibbs CR, Watson RD, Singh SP, et al. Management of pericardial effusion by drainage: a survey of 10 years' experience in a city centre general hospital serving a multiracial population. Postgrad Med J 2000;76:809-13.

9 Colombo A, Olson HG, Egan J, et al. Etiology and prognostic implications of a large pericardial effusion in men. Clin Cardiol 1988;11:389-94.

10 Loire R, Goineau P, Fareh S, et al. Apparently idiopathic chronic pericardial effusion. Long-term outcome in 71 cases. Arch Mal Coeur Vaiss 1996;89:835-41.

11 Long R, Younes M, Patton N, et al. Tuberculous pericarditis: long term outcome in patients who received medical therapy alone. Am Heart J 1989;117:1133-9.

12 Cherian G, Habashy AG, Uthaman B, et al. Detection and follow-up of mediastinal lymph node enlargement in tuberculous effusions using computed tomography. Am J Med 2003;114:319-22.

13 Fowler NO. Tuberculous pericarditis. JAMA 1991;266:99-103.

14 Strang JIG, Kakaza HHS, Gibson DG, et al. Controlled clinical trial of complete open surgical drainage and of prednisolone treatment of tuberculous pericardial effusion in the Transkei. Lancet 1988;ii:759-64.

15 Ortbals DW. Tuberculous pericarditis. Arch Intern Med 1979;139:231-4.

16 Eliskova M, Eliska O, Miller AJ. The lymphatic drainage of the parietal pericardium in man. Lymphology 1995;28:208-17.

17 Eliskova M, Eliska O, Miller AJ, et al. The efferent cardiac lymphatic pathways in the macaque monkey. Lymphology 1992:25:69-74.

18 Miller AJ, DeBoer A, Pick R, et al. The lymphatic drainage of the pericardial space in the dog. Lymphology 1988;21:227-33.

19 Pesola G, Teirstein AS, Goldman M. Sarcoidosis presenting with pericardial effusion. Sarcoidosis 1987:4:42-4.

20 Spodick DH. Tuberculous pericarditis. Arch Intern Med 1956;98:737-49.

21 Chitnis AS, Joshi VR, Cherian A, et al. Utility of scalene node biopsy in the diagnosis of pleuro- pericardial tuberculosis. J Assoc Physicians India 1974;22:805-8.

22 Light RW. Disorders of pleura mediastinum diaphragm. In: Isselbacher KJ, Braunwald E, Wilson JD, et al. Harrison's principles of internal medicine. New York: McGraw-Hill, 1994:1229-34.

23 Klein JS. The hila. In: Brant WE, Helms CA, eds. Fundamentals of diagnostic radiology. Baltimore: Williams and Wilkins, 1994:390-411.

24 Kobzik L. The lung. In: Cotran RS, Kumar V, Collins T, eds. Robbins pathologic basis of disease. Philadelphia: WB Saunders, 1999, 351, 697-755.

25 Rana BS, Jones RA, Simpson IA. Recurrent pericardial effusion: the value of polymerase chain reaction in the diagnosis of tuberculosis. Heart 1999;82:246-7.

26 Cegielski JP, Devlin BH, Morris AJ, et al. Comparison of PCR, culture, and histopathology for diagnosis of tuberculous pericarditis. J Clin Microbiol 1997; $35: 3254-7$.

27 Lee JH, Lee CW, Lee SG, et al. Comparison of polymerase chain reaction with adenosine deaminase activity in pericardial fluid for the diagnosis of tuberculous pericarditis. Am J Med 2002;113:519-21.
$28 \mathrm{Ng}$ TT, Strang Jl, Wilkins EG. Serodiagnosis of pericardial tuberculosis. $Q J$ Med 1995;88:317-20.

29 Dieli F, Sireci G, Ivanyi J, et al. Broad clonal heterogeneity of antigen-specific $\mathrm{CD} 4+\mathrm{T}$ cells localizing at the site of disease during tuberculosis. Immunol Lett 1999:69:311-15.

30 Burgess L, Reuter H, Carstens ME, et al. The use of adenosine deaminase and interferon gamma as diagnostic tools for tuberculous pericarditis. Chest 2002; 122:900-5

31 Komsuoglu B, Goldeli O, Kulan K, et al. The diagnostic and prognostic value of adenosine deaminase in tuberculous pericarditis. Eur Heart $J$ $1995 ; 16: 1126-30$

32 Aggeli C, Pitsavos C, Brili S, et al. Relevance of adenosine deaminase and lysozyme measurements in the diagnosis of tuberculous pericarditis. Cardiology 2000;94:81-5.

33 Lin DS, Tipton RE. Ga-67 cardiac uptake. Clin Nucl Med 1983;8:603-4.

34 Schmidt U, Rebarber IF. Tuberculous pericarditis identified with gallium-67 and indium-111 leukocyte imaging. Clin Nucl Med 1994;19:146-7.

35 Hugo-Hamman CT, Scher H, De Moor MM. Tuberculous pericarditis in children: a review of 44 cases. Pediatr Infect Dis 1994;13:13-18.

36 Gladych E, Goland S, Attali M, et al. Cardiac tamponade as a manifestation of tuberculosis. South Med J $2001 ; 94: 525-8$

37 Cegielski JP, Lwakatare J, Dukes CS, et al. Tuberculous pericarditis in Tanzanian patients with and without HIV infection. Tuber Lung Dis 1994;75:429-34.

38 Glazer GM, Gross BH, Quint LE, et al. Normal mediastinal lymph nodes: number and size according to American Thoracic Society mapping. Am J Roentgenol 1985; 144:261-5.

39 Ingram CE, Belli AM, Lewars MD, et al. Normal lymph node size in the mediastinum: a retrospective study in two patient groups. Clin Radio 1989:40:35-9.

40 Liu PY, Li YH, Tsai WC, et al. Usefulness of echocardiographic intrapericardial abnormalities in the diagnosis of tuberculous pericardial effusion. Am J Cardiol 2001;87:1133-5.

41 Strang Jl. Tuberculos pericarditis in Transkei. Clin Cardiol 1984;7:667-70.

42 Agner RC, Gallis HA. Pericarditis. Differential diagnostic considerations. Arch Intern Med 1979;139:407-12

43 Strang JIG. Rapid resolution of tuberculous pericardial effusions with high dose prednisone and anti-tuberculous drugs. J Infect 1994;28:251-4.

\section{ANSWERS}

(1) Tuberculosis, viral, and chronic idiopathic. ${ }^{8-10} 1242$ (2) From infected mediastinal lymph nodes. ${ }^{75}$ (3) Hilar group..$^{12}$

(4) No. Not seen on plain chest radiograph. ${ }^{12}(5)$ True. $^{14}$ (6)

Excellent. ${ }^{11}{ }^{12}$ (7) True. $^{235}$ 